\title{
Deletion of all Cochliobolus heterostrophus Monofunctional Catalase-Encoding Genes Reveals a Role for One in Sensitivity to Oxidative Stress but None with a Role in Virulence
}

\author{
Barbara Robbertse, ${ }^{1}$ O. C. Yoder, ${ }^{1}$ Anita Nguyen, ${ }^{1}$ Conrad L. Schoch, ${ }^{2}$ and B. Gillian Turgeon ${ }^{2}$ \\ ${ }^{1}$ Former Torrey Mesa Research Institute, 3115 Merryfield Row, San Diego, CA 92122, U.S.A.; ${ }^{2}$ Department of Plant \\ Pathology, Cornell University, Ithaca, NY 14853, U.S.A.
}

Submitted 14 April 2003. Accepted 19 June 2003.

\begin{abstract}
The genome of the maize pathogen Cochliobolus heterostrophus encodes three unlinked monofunctional catalaseencoding $(C A T)$ genes that singly or in combination could offer protection against the harmful effects of oxidative stress. Phylogenetic analysis placed the CAT2 and CAT3 proteins in a cluster with large subunit catalases (CAT3 has a secretory signal sequence and was grouped with known secreted catalases), whereas CAT1 clustered with small subunit catalases. Single, double, and triple cat mutants were created and screened for sensitivity to hydrogen peroxide and altered virulence on maize. All mutants deficient in $C A T 3$ had enhanced sensitivity to hydrogen peroxide, as compared with wild type or with mutants deficient in CAT1, CAT2, or both. All catalase-deficient mutants had normal virulence to maize. Thus, the secreted CAT3 protein protects the fungus from oxidative stress during vegetative growth, but members of this enzyme family, alone or in combination, are not essential for virulence.
\end{abstract}

Additional keywords: fungi, genome sequence, pathogenicity.

When under attack, plant hosts of pathogenic fungi release reactive oxygen species (ROS), presumably as a defense mechanism (Shim and Dunkle 2002; Wang et al. 1996). Production of ROS involves reduction of molecular oxygen to form superoxide anions $\left(\mathrm{O}_{2}^{-}\right)$, followed by spontaneous dismutation of the superoxide anion to yield hydrogen peroxide and oxygen. Hydroxyl radicals $\left(\mathrm{OH}^{-}\right)$develop via metal reduction in a hydrogen peroxide-dependent manner. Hydroxyl radicals are the major cause of cellular damage, which involves lipid peroxidation, enzyme inactivation, and degradation of nucleic acids (Thompson et al. 1987). These activities can be effective against the plant cells that produce ROS and can also be di-

Corresponding author: B. Gillian Turgeon; 334 Plant Science Building, Cornell University; E-mail: bgt1@ cornell.edu; Telephone: 607-2547458; Fax number: 607-255-8835.

B. G. Turgeon and O. C. Yoder codirected this work.

This work was done while B. G. Turgeon was on a leave of absence at Torrey Mesa Research Institute.

Current address for B. Robbertse and C. L. Schoch: Department of Botany and Plant Pathology, Oregon State University, Corvallis, OR 97331, U.S.A.

Current address for O. C. Yoder: Diversa Corporation, 4955 Directors Place, San Diego, CA 92121, U.S.A. rected against invading pathogens (Keppler et al. 1989). Production of ROS is well documented in microbe-dicot interactions (Bestwick et al. 1998) and has also been observed after infection of monocots, such as barley and rice (Kumar et al. 2001; Mehdy 1994).

Many fungi produce catalases and other enzymes that help them survive stress caused by $\mathrm{ROS}$, e.g., $\mathrm{O}_{2}^{-}, \mathrm{OH}^{-}$, and hydrogen peroxide (Schouten et al. 2002). Although enzymes such as superoxide dismutases, peroxidases, and catalases are wellknown ROS scavengers, their importance during infection and survival of a fungus in its host remains uncertain. There is evidence that hydrogen peroxide and catalase activity are associated with infection of maize by the fungus Exserohilum turcicum (Keissar et al. 2002).

In this study, the potential contribution of all monofunctional catalases to virulence of Cochliobolus heterostrophus on maize was investigated. $C$. heterostrophus belongs to the Pleosporales, a sister taxon of E. turcicum, and causes Southern Corn Leaf Blight (Drechsler 1925), whereas E. turcicum causes northern leaf blight. Our investigation was made possible by availability of the $C$. heterostrophus genome sequence, which was sequenced by Celera Genomics for Torrey Mesa Research Institute (TMRI)/Syngenta, and the fact that targeted mutations are generated efficiently in this organism (Catlett et al. 2003; Turgeon et al. 1987; Wirsel et al. 1996). Furthermore, it is easy to do crosses in the laboratory with this fungus. Three monofunctional catalase genes were identified in the genome, single, double, and triple deletion mutants were generated, and their phenotypes determined. We found that deletion of one of these genes enhanced oxidative stress sensitivity but did not affect virulence to maize.

\section{RESULTS}

Structures of catalase genes and deduced amino-acid sequences.

Three monofunctional catalase $(C A T)$ genes, designated $C A T 1, C A T 2$, and CAT3, were identified in the $C$. heterostrophus genome, using a tblastn query with protein sequences of several known catalases. ReBLAST of the $C$. heterostrophus genome with the putative $C$. heterostrophus catalases did not reveal additional ones. The putative $C A T$ coding sequences and corresponding predicted introns are: $C A T 1,1,641 \mathrm{bp}$ with one intron (72 bp); CAT2, 2,220 bp with one intron (54 bp); and CAT3, 2,190 bp with six introns $(53,48,62,55,54$, and $55 \mathrm{bp})$. Proteins deduced from the $C A T 1, C A T 2$, and $C A T 3$ open reading 
frames were 547, 740, and 730 amino acids long, with predicted molecular masses (and isoelectric points) of 63 (5.97), 83.2 (5.78), and $79.9 \mathrm{kDa}$ (5.94), respectively. All three catalases have both the proximal heme-ligand and active site PROSITE signature motif sequences that distinguish true catalases.

To predict functions of the $C$. heterostrophus catalases, phylogenetic analyses were conducted (data not shown). Both maximum likelihood analysis of amino acid sequences based on Bayesian inference and maximum parsimony analysis showed two well-supported clades, corresponding to small (50 to $65 \mathrm{kDa}$ ) and large (approximately $80 \mathrm{kDa}$ ) subunit catalases (Klotz et al. 1997).
Distinct clades representing the three groups of catalases (Groups I, II, and III) defined in previous studies (Bussink and Oliver 2001; Klotz et al. 1997) were also evident, as was strong support for distinct secreted and nonsecreted subgroups of Group II. Fungal catalases fell in Groups II and III only, as found previously (Klotz et al. 1997). CAT2 (Group II) clustered with nonsecreted catalases and is closely related to CAT2 of Cladosporium fulvum (Bussink and Oliver 2001). CAT3 (Group II) clustered with the secreted catalases. Consistent with this, a signal peptide leader sequence with a cleavage site between amino acid positions 19 and 20 in the CAT3 protein is predicted (Nielsen et al. 1997). CAT1 is a putative small sub-

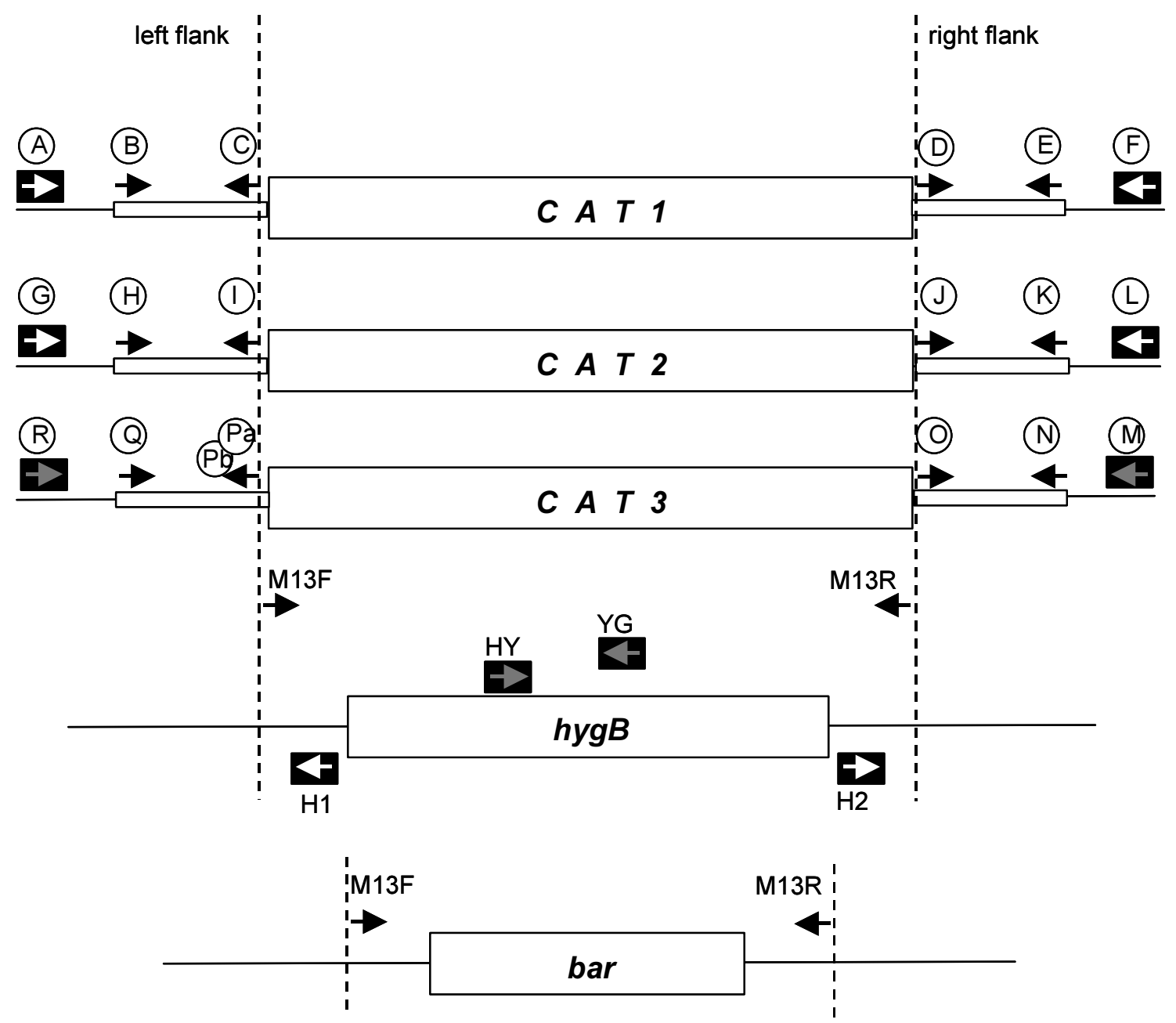

Fig. 1. Positions (indicated by letters) of primers used for the design of single and triple deletion constructs and analysis of catalase-encoding (CAT) gene deletions. Black arrows indicate primers used to construct deletions only, gray arrows indicate primers used for the design of deletion constructs and analysis of deletions, white arrows indicate primers used to analyze gene deletions only, and dotted vertical lines indicate borders of gene replacement. Sizes of flanks used for deletion ranged from 327 to $461 \mathrm{bp}$. On the diagram, 5 to $3^{\prime}$ orientation of $C A T 1$, CAT3, hygB, and bar is from right to left. CAT2 5 to $3^{\prime}$ orientation is left to right. Note orientation of $h y g B$ is reversed in the CAT2 deletion strain, as compared with the CAT1 and CAT3 mutants.

Table 1. Primer combinations (primer position in brackets) used to check integration events in single (a), double (b), and triple (c) catalase mutants

\begin{tabular}{llcc}
\hline Gene deletion & \multicolumn{1}{c}{ Primer combinations } & $\begin{array}{c}\text { Size (kb) of product expected } \\
\text { with DNA from mutants }\end{array}$ & $\begin{array}{c}\text { Size (kb) of product expected } \\
\text { with wild-type DNA }\end{array}$ \\
\hline CAT1 ab & GCAT1-P0 (A) and NCL38 (YG) & 2.9 & none \\
& GCAT1-P5 (F) and NCL37 (HY) & 2.1 & none \\
CAT2ab & GCAT2-P0 (G) and NCL38 (YG) & 2.8 & none \\
& GCAT2-P5 (L) and NCL37 (HY) & 2.25 & none \\
CAT3 ab & GCAT3-P0 (M) and H2 & 1.4 & none \\
& GCAT3-P5 (R) and H1 & 1.3 & 3.8 \\
CAT1 c & GCAT1-P0 (A) and GCAT1-P5 (F) & 4.5 & 4.4 \\
CAT2 c & GCAT2-P0 (G) and GCAT2-P5 (L) & 4.6 & 5.1 \\
CAT3 c & GCAT3-P0 (M) and GCAT3-P5 (R) & 3.9 & \\
\hline
\end{tabular}


unit catalase that clustered in Group III, distinct from the large subunit catalases in Group II. All predicted and known peroxisomal catalases cluster in Group III. However, C. heterostrophus CAT1 does not contain signature motifs proposed for peroxisomal catalases, (Gould et al. 1989; Swinkels et al. 1991).

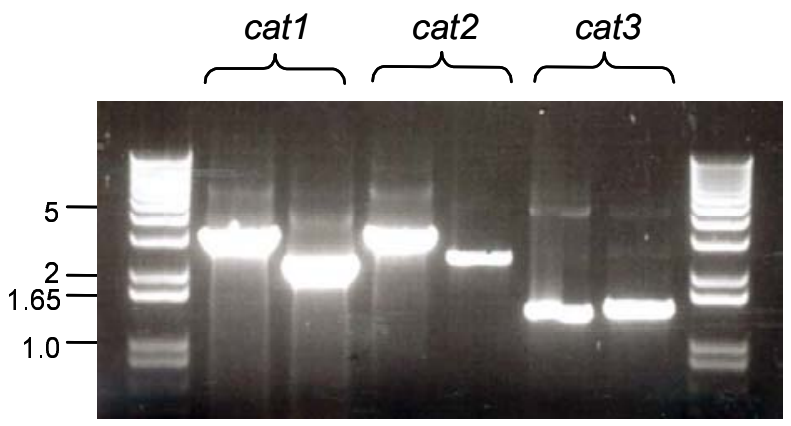

Fig. 2. Polymerase chain reaction confirmation of single catalase gene deletions. Template DNA: cat1 = D.C5.cat1-1B, cat2 = D.C5.cat2-3A, $c a t 3=$ D.C5.cat3-1B. For $c a t 1$ and cat3, left and right lanes correspond to checks of targeted deletion of the $3^{\prime}$ and $5^{\prime}$ ends of the genes, respectively; for cat2, left and right lanes correspond to checks of targeted deletion of the $5^{\prime}$ and $3^{\prime}$ ends of the gene, respectively. Outside lanes, 1 $\mathrm{kb}$ plus DNA ladder. Sizes are in $\mathrm{kb}$.
Confirmation of single, double, and triple cat deletions.

Figure 1 is a diagram of the CAT gene-deletion constructs and the locations of primers used to make and confirm deletions. Polymerase chain reaction (PCR) product sizes expected when DNA from wild-type vs. deletion mutants is used as template are listed in Table 1. PCR analysis using DNA from transformants carrying putative single, double (Figs. 2 and 3), or triple CAT deletions (Fig. 4) confirmed homologous integration of the selectable marker at the target site in each case.

\section{Sensitivity of single, double, and triple cat mutants to hydrogen peroxide.}

Growth rates of wild-type strains (C5 and N21-R-24) and cat 1 and cat 2 mutants were not significantly different $(P>$ 0.05 ) on hydrogen peroxide-amended vs. nonamended medium (Fig. 5). Cat3 mutant strains, however, grew normally on nonamended plates but significantly $(P<0.05)$ slower on amended medium (Fig. 5). The hydrogen peroxide-sensitive phenotype cosegregated with resistance to hygromycin B (Fig. 5), confirming that sensitivity is due to deletion of CAT3. Growth rates of cat 1;cat3 and cat 2 ;cat 3 double mutants or cat 1;cat $2 ;$ cat 3 triple mutant were also significantly reduced $(P<0.001)$ on hydrogen peroxide, compared with that of wild-type strains, whereas cat 1 ; cat 2 double mutants were not $(P>0.05)$. Mutations in $C A T 1$ or $C A T 2$, or both, did not enhance sensitivity of

A

cat2

cat1
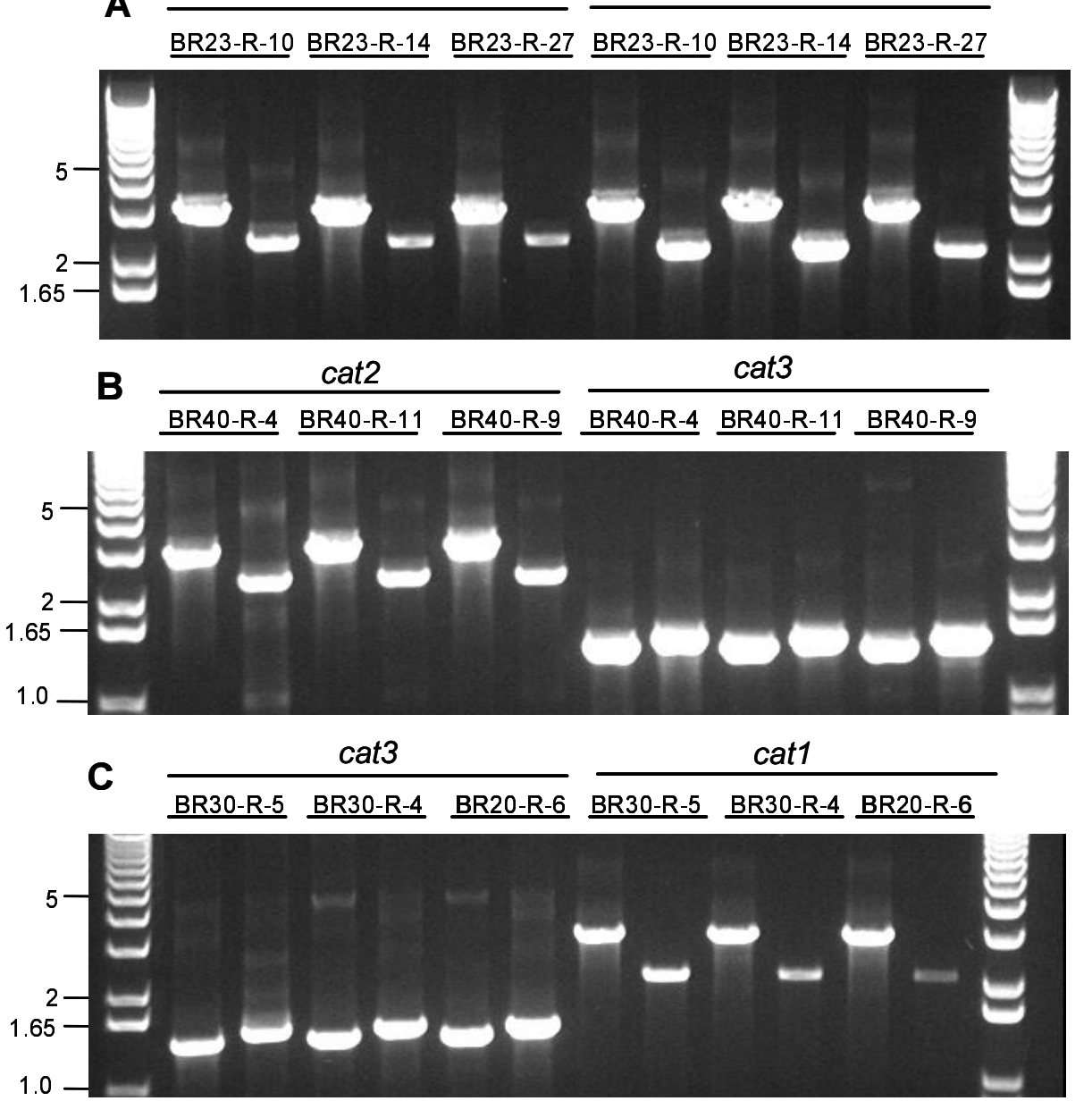

Fig. 3. Polymerase chain reaction analysis of double catalase mutants to confirm deletion of pairs of $C A T$ genes. A, cat 1 ;cat 2 double mutants, B, cat 2 ;cat 3 double mutants, and $\mathbf{C}$, cat 1;cat3 double mutants. Strains used for template DNA are indicated at the top of each panel. Primer combinations are listed in Table 1. For cat 1 and cat 3 , left and right lanes correspond to checks of targeted deletion of the $3^{\prime}$ and $5^{\prime}$ ends of the gene, respectively; for cat 2 , left and right lanes correspond to checks of targeted deletion of the $5^{\prime}$ and $3^{\prime}$ ends of the gene, respectively. Expected product sizes are listed in Table 1. Outside lanes, $1 \mathrm{~kb}$ plus DNA ladder. Note that each panel represents three independent mutants. The left part of each panel is an assessment of deletion of one of the two $C A T$ genes, the right side is the same template DNA assessed for the second CAT gene. 
cat3 mutants (Fig. 5). Thus, CAT3 alone contributes to protection of $C$. heterostrophus against oxidative stress during vegetative growth.

\section{Virulence of single, double, and triple cat mutants.}

Symptoms caused by cat 1 , cat 2 , or cat 3 mutants or double or triple cat mutants were similar to those caused by wild-type strains of $C$. heterostrophus on maize (Fig. 6A and B). These results came from analysis of progeny of crosses between purified mutant transformants and wild-type testers. Primary cat 2 transformant D.C5.Cat2-3A, after purification by single sporing, was exceptional in that it caused smaller and fewer lesions on maize seedlings than did wild type. However, this transformant also had slow growth on nonamended minimal medium $(\mathrm{MM})$ and the reduced virulence phenotype did not cosegregate with resistance to hygromycin B in progeny of crosses to wild type. Therefore, reduced virulence was not associated with cat 2 and was eliminated in progeny of a cross.

\section{DISCUSSION}

Results of this study indicate that $C$. heterostrophus monofunctional catalases are not required for normal virulence of the fungus to maize. This conclusion could be drawn because we were able to extract all putative monofunctional catalase genes from the $C$. heterostrophus genome sequence and delete them all by taking advantage of the highly efficient system for gene deletion via homologous recombination in this fungus. In addition, the ease of doing crosses in the laboratory allowed us to quickly construct double mutants. These resources positioned us to assay the virulence phenotype of single, double, and triple monofunctional catalase mutants on maize.

Several previous studies of catalase-deficient fungal mutants and their virulence to plants have been reported. In all of these studies, only single gene mutations were evaluated (Bussink and Oliver 2001; Calera et al. 1997; Garre et al. 1998; Schouten et al. 2002; Wysong et al. 1998). Among the CAT genes studied was that of Candida albicans, which, when mutated, caused reduced virulence in an intravenous infection model of disseminated candidiases in mouse (Wysong et al. 1998). Three studies involving plant-fungal pathogen systems yielded no catalase mutants that caused reduced virulence. These included $C$. fulvum on tomato (two catalases reported, Bussink and Oliver 2001), Claviceps purpurea on rye (one catalase reported, Garre et al. 1998) and Botrytis cinerea on to-

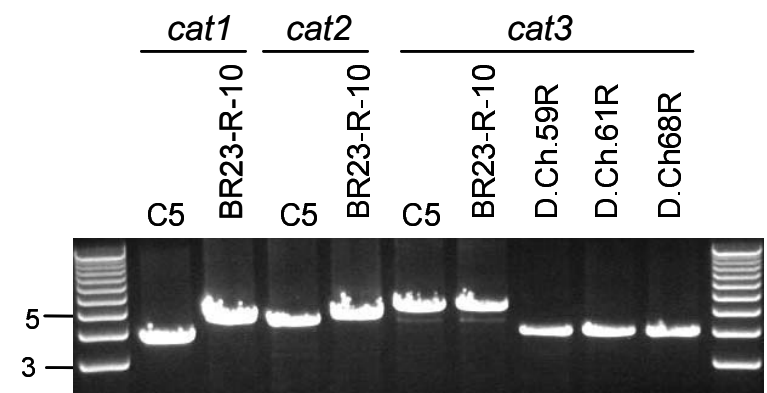

Fig. 4. Polymerase chain reaction confirmation of deletion of CAT3 in cat1;cat2 double mutant BR23-R-10. The cat1 (assayed with primer combination GCAT1-P0/ GCAT1-P5) and cat2 (assayed with primer combination GCAT2-P0/ GCAT2-P5) controls (from left, lanes 2 to 5) confirm that this pair of $C A T$ genes is deleted in BR23-R-10. Lanes 6 and 7, cat3 (assayed with primer combination GCAT3-P0/GCAT3-P5) controls, C5 and BR23-R-10, which are wild type for CAT3 and thus yield the wild-type band. Lanes 8 to 10, triple catalase mutants D.Ch.59R, D.Ch61R, and D.Ch68R are deleted for cat3 (assayed with primer combination GCAT3-P0/ GCAT3-P5). Outside lanes, $1 \mathrm{~kb}$ plus DNA ladder. mato and bean (two catalases reported, Schouten et al. 2002). One study with Aspergillus fumigatus (Calera et al. 1997) investigated the effect of deletion of one of two catalases on pathogenicity in a mouse model of aspergillosis. No differences were observed between the cat 1 mutant and its parental CAT1 strain.

Our observation of normal virulence in monofunctional catalase-deficient mutants does not imply that resistance to oxidative stress is unimportant for fungal virulence, since there are other enzymes known to be involved in detoxification of ROS. In fact, three different gene families that encode catalases able to detoxify hydrogen peroxide are known: i) manganese catalases (Barynin et al. 1986), ii) catalase peroxidases (Fraaije et al. 1996), and (iii) the true (monofunctional) catalases. This paper focused on true catalases, which are homotetrameric, hemecontaining enzymes found in eukaryotes and prokaryotes (Klotz et al. 1997; Schonbaum and Chance 1976). Catalase peroxidases are also found in lower eukaryotes, but manganese catalases have been reported only in prokaryotes. Related to this, a reported fourth true catalase in A. nidulans was reannotated as a catalase-peroxidase (Kawasaki and Aguirre 2001; Scherer et al. 2002). A tblastn search of the C. heterostrophus genome, using the protein sequence of the A. nidulans catalaseperoxidase as a query, produced one strong hit. A catalase previously reported to be a virulence factor for tumorigenesis in the Agrobacterium tumefaciens-rye interaction also appears to be a catalase-peroxidase, not a true catalase. It lacks the two distinct PROSITE motifs found in monofunctional catalases and is homologous to the same $C$. heterostrophus hit identified in the query with the $A$. nidulans catalase-peroxidase. The $C$. heterostrophus catalase-peroxidase could have compensated for a possible virulence function of monofunctional catalases in C. heterostrophus. A tblastn search of the C. heterostrophus genome for the presence of manganese catalases did not yield any high hits, as would be predicted given their known prokaryote distribution.

The catalase triple mutant of $C$. heterostrophus had normal virulence, despite hyphae being clearly sensitive to hydrogen peroxide. This observation raises several questions to be addressed in the future. Were plant-produced hydrogen peroxide levels sufficiently high under our experimental conditions to cause inhibition of fungal growth during infection? If so, was the hydrogen peroxide in contact with the fungus? Was the

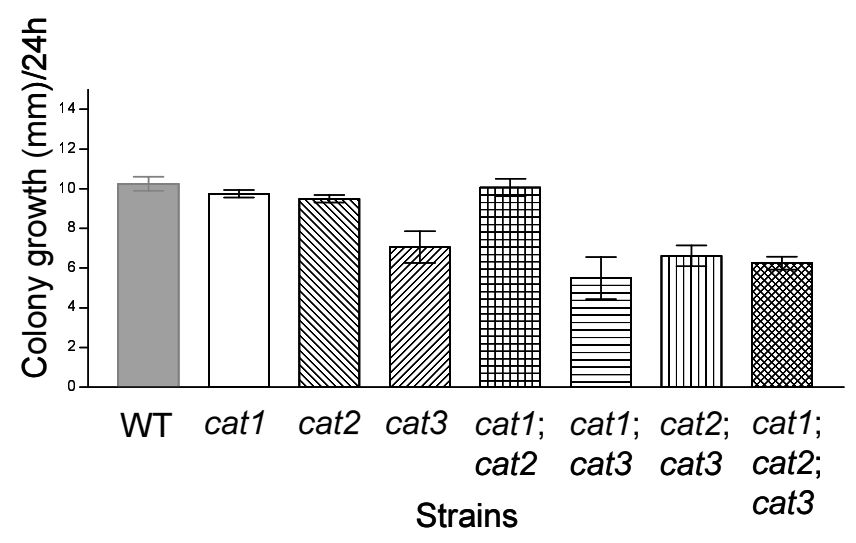

Fig. 5. Growth of wild-type and cat1, cat 2 , and cat 3 deletion progeny in $24 \mathrm{~h}$, calculated as a change in colony diameter on $5 \mathrm{mM} \mathrm{H}_{2} \mathrm{O}_{2}$-amended minimal medium. Strains are wild-type strains (C5 and N21-R-24) and catalase deletion mutants, cat1 (BR14-1-4 and BR14-R-1), cat2 (BR122-2 and BR12-2-3), cat3 (BR11-R-2 and BR11-R-6), cat1; cat2 (BR23-R10 and BR23-R-27), cat1;cat3 (BR40-R-4 and BR40-R-11), cat2;cat3 (BR30-R-4 and BR30-R-5), and cat1;cat2;cat3 (D.Ch.59R, D.Ch61R, and D.Ch68R). Bar = standard deviation. 
plant test sensitive enough to detect small changes in fungal virulence? Given these open questions, the role of fungal tolerance to ROS in virulence remains unresolved. Future studies should include deletion of all genes encoding enzymes that use ROS as substrates.

\section{MATERIALS AND METHODS}

Strains, media, crosses, and transformation.

C. heterostrophus culture media, growth conditions, crossing (Leach et al. 1982), and transformation procedures (Straubinger et al. 1990; Turgeon et al. 1987) were described previously. Strains used or constructed in this study are listed in Table 2. Transformations were conducted in strain C5, using the gene for resistance to hygromycin $\mathrm{B}$ (hygB) as a selectable marker (Turgeon et al. 1987) or, in strain BR23-R10 , using the gene for resistance to bialaphos (bar) as a se- lectable marker (Straubinger et al. 1990). All transformants were purified by isolation of single conidia on complete medium $(\mathrm{CM})$ without salts amended with $50 \mu \mathrm{g}$ hygromycin B per ml (Invitrogen, San Diego, CA, U.S.A.). Strains were grown on CM with xylose (CMX) (Tzeng et al. 1992) at $22^{\circ} \mathrm{C}$ under black lights (Sylvania Inc., Danvers, MA, U.S.A.) for conidiation. Purified transformants were crossed to strain N21-R-24 (Table 2) for progeny tests.

Double mutants were created by crossing single mutants in all possible combinations (Table 2). Ascospores were transferred to CMX plates, after which mycelium from germinated spores was transferred to selective medium to determine hygromycin B sensitivity. Triple mutant strains D.Ch.59R, D.Ch.61R, and DCh.68R were created by deletion of CAT3 in cat1; cat2 double mutant BR23-R-10. Transformants were purified by isolation of single conidia on MM amended with $75 \mu \mathrm{g}$ of bialaphos per ml (Meiji Seika Kaisha, Tokyo). Single-spored
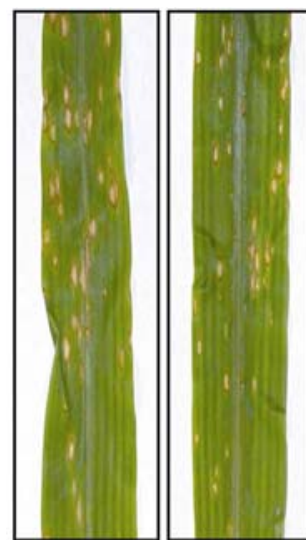

C5 N21-R-24 WT
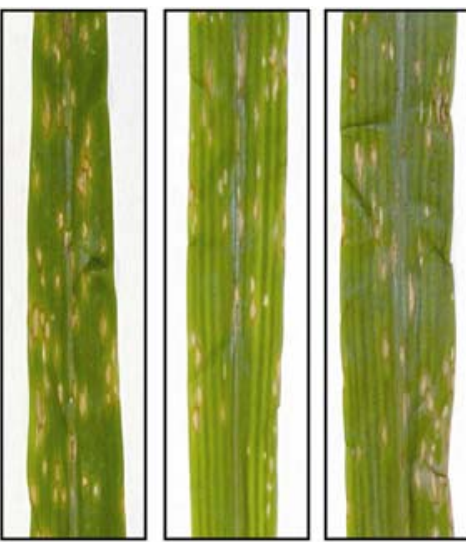

BR23-R-10 BR23-R-14

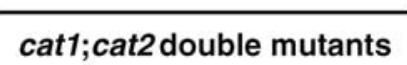

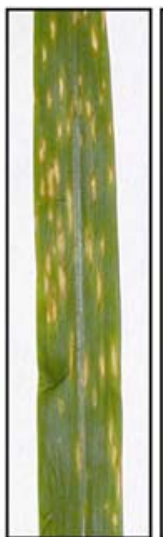
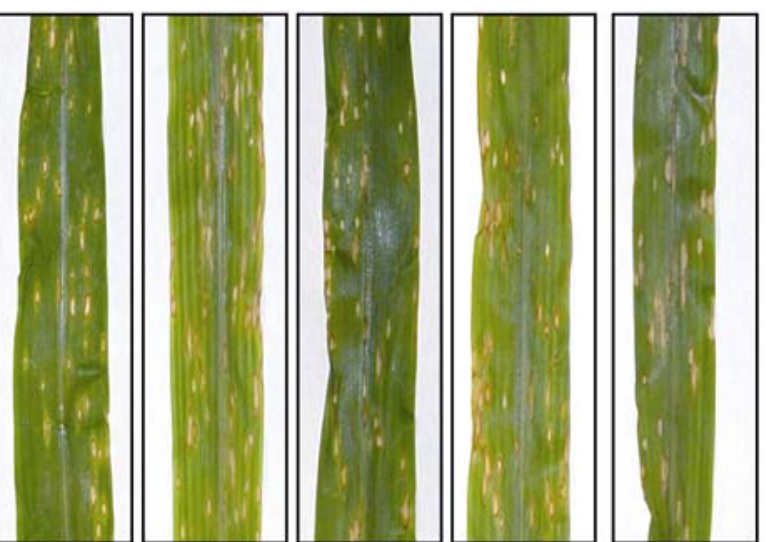

BR20-R-6

BR30-R-4

BR30-R-5

cat1; cat3 double mutants

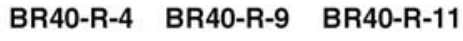

cat2;cat3 double mutants
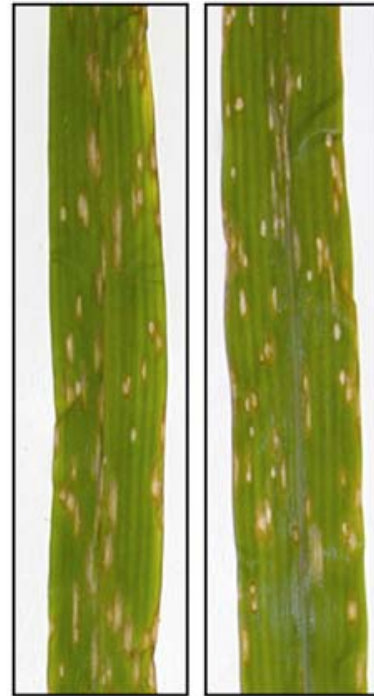

C5

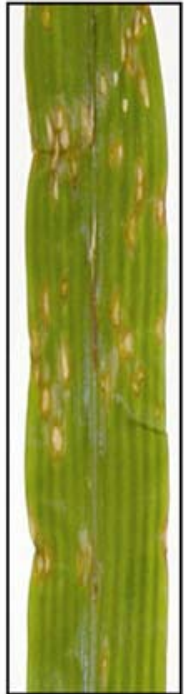

BR23-R-10

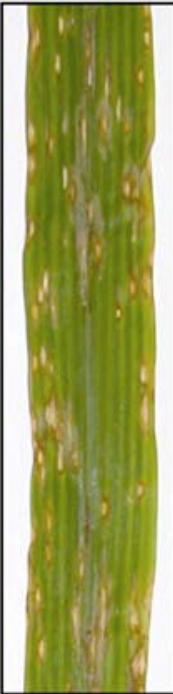

D.Ch.64S

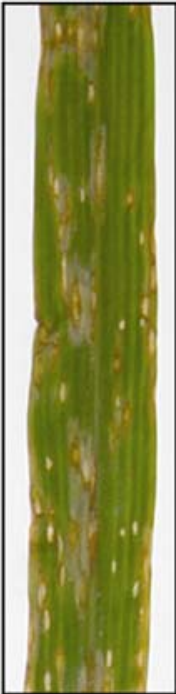

D.Ch.57S

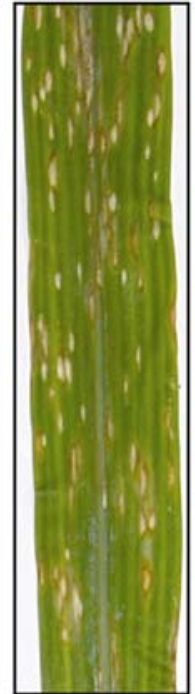

D.Ch.67S

WT

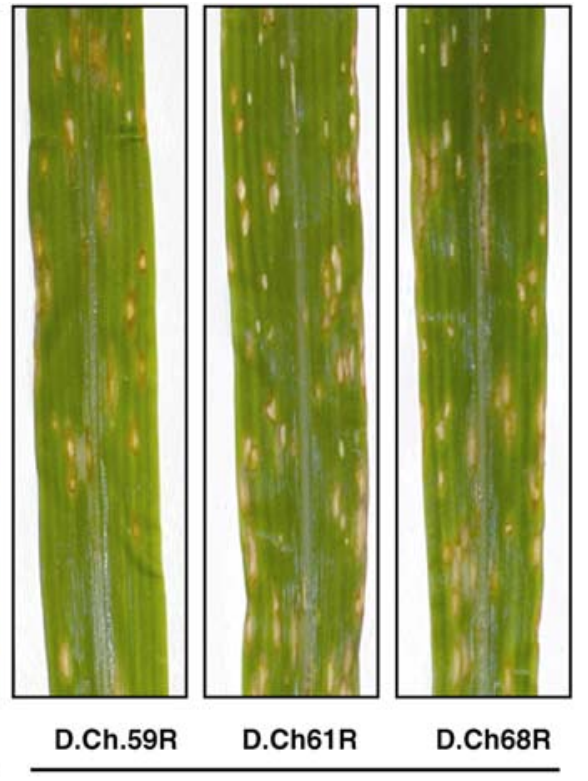

cat1;cat2;cat3 triple mutants

Fig. 6. Virulence assays. Top, Lesions on corn leaves caused by wild-type strains (C5 and N21-R-24) and cat1;cat2, cat1;cat3, and cat2;cat3 double mutants. No differences in quantity or quality of lesions were observed. Bottom, Lesions on W64-N-corn seedlings caused by wild type (C5 and N21-R24), cat 1;cat 2 double mutants, and cat 1;cat2;cat3 triple mutants. All are like wild type, indicating than none of these catalases alone or in combination is essential for virulence. BR23-R-10 is the cat1;cat2 progenitor strain in which cat3 was deleted. D.Ch.64S, D.Ch.57S, and D.Ch.67S are cat1;cat2;CAT3 strains resulting from the purification of transgenic heterokaryons. 
bialaphos-sensitive double mutant strains D.Ch.57S, D.Ch.64S, and D.Ch.67S were also obtained during single sporing of the primary bialaphos-resistant putative triple mutant, indicating that the original transformant was a heterokaryon, consisting of both bialaphos-resistant and sensitive nuclei. These were retained for comparison with bialaphos-resistant triple mutants in virulence assays (Table 2, Fig. 6B). Strains were stored at $-80^{\circ} \mathrm{C}$ in $25 \%$ glycerol and were retrieved from storage prior to each experiment.

\section{DNA isolation and PCR.}

Fungal genomic DNA was isolated using a DNeasy Plant mini or 96-well kit (Qiagen, Valencia, CA, U.S.A.). PCR amplification was performed, using either Hotstart master mix (Qiagen) or Expand polymerase (Roche. Indianapolis, IN, U.S.A.). The PCR program used with Hotstart master mix was as follows: step $1,95^{\circ} \mathrm{C}, 15 \mathrm{~min}$; step $2,96^{\circ} \mathrm{C}, 30 \mathrm{~s}$; step 3 , $54^{\circ} \mathrm{C}, 30 \mathrm{~s}$; and step $4,72^{\circ} \mathrm{C}, 1 \mathrm{~min}$ per kb. Steps 2 to 4 were repeated 36 times. The PCR program used with Expand was as follows: step $1,94^{\circ} \mathrm{C}, 2 \mathrm{~min}$; step $2,94^{\circ} \mathrm{C}, 30 \mathrm{~s}$; step $3,54^{\circ} \mathrm{C}$, $30 \mathrm{~s}$; step $4,68^{\circ} \mathrm{C}, 3.5 \mathrm{~min}$, and step $5,72^{\circ} \mathrm{C}$ for $10 \mathrm{~min}$. Steps 2 to 4 were repeated 30 times.
Identification of catalases

using comparative molecular and phylogenetic analyses.

Amino acid sequences of known catalase genes were used to query (tblastn; Altschul et al. 1997) the C. heterostrophus genome sequence generated by Celera Genomics for TMRI/Syngenta to identify all monofunctional catalases. Open reading frames were annotated using Artemis (Rutherford et al. 2000). The CAT1, CAT2, and CAT3 sequences were assigned GenBank accession numbers AY369262, AY369263, and AY369264, respectively. Protein sequences of selected catalases in GenBank were aligned with the $C$. heterostrophus sequences using T-Coffee (Notredame et al. 2000). Alignments were assessed manually and were improved, in certain cases, using Genedoc (Nicholas et al. 1997). For phylogenetic analysis, a maximum likelihood analysis using Bayesian inference was performed with the program MrBayes (Huelsenbeck and Ronquist 2001). MrBayes was run with the following parameters: a general model of DNA distribution (GTR) with gammadistributed rate variation across sites. A Markov chain Monte Carlo analysis with four chains was run for 1,000,000 generations, with a sample frequency of 100. This yielded 10,000 trees, of which 200 were discarded. For additional comparison,

Table 2. Cochliobolus heterostrophus strains used in this study

\begin{tabular}{|c|c|c|}
\hline Name & Genotype & Source \\
\hline N21-R-24 & WT; MAT1-2 & $\begin{array}{l}\text { N21-R-24 = progeny of N3-4-2 }(M A T 1-2) \times \mathrm{N} 3-4-4(M A T 1-1) \text { cross } \\
\text { N3-4-2 }=\text { progeny of N2-2-6 }(M A T 1-2) \times \mathrm{C} 5 \text { cross } \\
\text { N2-2- } 6=\text { progeny of } \mathrm{C} 4 \times \mathrm{C} 5 \text { cross }\end{array}$ \\
\hline C5 & WT; MAT1-1 & ATCC number 48331 (Leach et al. 1982) \\
\hline $\mathrm{C} 4$ & WT; MAT1-2 & ATCC number 48332 (Leach et al. 1982) \\
\hline D.C5.cat1-1B & cat1;MAT1-1;hygB & C5 carrying a $C A T 1$ deletion \\
\hline D.C5.cat2-3A & cat $2 ;$ MAT1-1;hygB & C 5 carrying a $C A T 2$ deletion \\
\hline D.C5.cat3-1B & cat $3 ; M A T 1-1 ;$ hygB & C5 carrying a CAT3 deletion \\
\hline BR14-1-4 & cat1;hygB;MAT1-1 & Progeny of a N21-R-24 $\times$ D.C 5 .cat $1-1 B$ cross \\
\hline BR14-R-1 & cat1;hygB;MAT1-1 & Progeny of a N21-R-24 $\times$ D.C5.cat $1-1 B$ cross \\
\hline BR14-R-4 & cat1; hyg B;MAT1-2 & Progeny of a N21-R-24 $\times$ D.C5.cat $1-1 B$ cross \\
\hline BR14-1-1 & cat $1 ;$ hygB $; M A T 1-2$ & Progeny of a N21-R-24 $\times$ D.C5.cat $1-1 B$ cross \\
\hline BR14-R-8 & WT & Progeny of a N21-R-24 $\times$ D.C5.cat $1-1 B$ cross \\
\hline BR14-R-11 & WT & Progeny of a N21-R-24 $\times$ D.C5.cat $1-1 B$ cross \\
\hline BR14-R-12 & WT & Progeny of a N21-R-24 $\times$ D.C5.cat1-1B cross \\
\hline BR14-R-14 & WT & Progeny of a N21-R-24 $\times$ D.C5.cat $1-1 B$ cross \\
\hline BR12-2-1 & cat $2 ; \operatorname{hyg} B$ & Progeny of a N21-R-24 $\times$ D.C5.cat2-3A cross \\
\hline BR12-2-2 & cat $2 ;$ hyg B $; M A T 1-2$ & Progeny of a N21-R-24 $\times$ D.C5.cat2-3A cross \\
\hline BR12-2-3 & cat $2 ;$ hyg B;MAT1-1 & Progeny of a N21-R-24 $\times$ D.C5.cat2-3A cross \\
\hline BR12-R-3 & cat $2 ;$ hyg $B$ & Progeny of a N21-R-24 $\times$ D.C5.cat2-3A cross \\
\hline BR12-1-1 & WT & Progeny of a N21-R-24 $\times$ D.C5.cat2-3A cross \\
\hline BR12-1-4 & WT & Progeny of a N21-R-24 × D.C5.cat2-3A cross \\
\hline BR12-R-2 & WT & Progeny of a N21-R-24 $\times$ D.C5.cat2-3A cross \\
\hline BR12-R-4 & WT & Progeny of a N21-R-24 × D.C5.cat2-3A cross \\
\hline BR11-R-2 & cat $3 ;$ hyg B;MAT1-2 & Progeny of a N21-R- $24 \times$ D.C5.Cat3-1B cross \\
\hline BR11-R-6 & cat $3 ;$ hyg B;MAT1-1 & Progeny of a N21-R-24 $\times$ D.C 5. cat $3-1 B$ cross \\
\hline BR11-R-7 & cat $3 ;$ hygB $; M A T 1-1$ & Progeny of a N21-R-24 $\times$ D.C5.Cat3-1B cross \\
\hline BR11-3-1 & cat $3 ;$ hyg $B ; M A T 1-1$ & Progeny of a N21-R- $24 \times$ D.C5.catt3-1B cross \\
\hline BR11-R-1 & WT & Progeny of a N21-R- $24 \times$ D.C5. cat3-1B cross \\
\hline BR11-R-4 & WT & Progeny of a N21-R- $24 \times$ D.C5.cat $3-1 B$ cross \\
\hline BR11-R-5 & WT & Progeny of a N21-R-24 $\times$ D.C5.cat3-1B cross \\
\hline BR11-2-3 & WT & Progeny of a N21-R-24 $\times$ D.C5.cat $3-1 B$ cross \\
\hline BR23-R-10 & cat $1 ;$ cat $2 ;$ hyg $B$ & Progeny of a BR14-R-1 $\times$ BR12-2-2 cross \\
\hline BR23-R-14 & cat 1 ; cat $2 ;$ hygB & Progeny of a BR14-R-1 $\times$ BR12-2-2 cross \\
\hline BR23-R-27 & cat 1 ; cat $2 ;$ hyg $B$ & Progeny of a BR14-R-1 $\times$ BR12-2-2 cross \\
\hline BR20-R-6 & cat 1 ; cat $3 ;$ hyg $B$ & Progeny of a BR14-R- $4 \times$ BR11-R- 6 cross \\
\hline BR30-R-4 & cat 1 ; cat $3 ;$ hyg $B$ & Progeny of a BR14-R- $4 \times$ BR11-R- 6 cross \\
\hline BR30-R-5 & cat 1 ; cat $3 ;$ hyg $B$ & Progeny of a BR14-R- $4 \times$ BR $11-R-6$ cross \\
\hline BR40-R-4 & cat 2 ; cat $3 ;$ hyg $B$ & Progeny of a BR12-2-2 $\times$ BR11-3-1 cross \\
\hline BR40-R-9 & cat 2 ; cat $3 ;$ hyg $B$ & Progeny of a BR12-2-2 $\times$ BR11-3-1 cross \\
\hline BR40-R-11 & cat 2 ; cat $3 ;$ hygB & Progeny of a BR12-2-2 $\times$ BR11-3-1 cross \\
\hline D.Ch.59R & cat $1 ;$ cat $2 ;$ cat $3 ;$ hyg $B ;$ bar & BR23-R-10 carrying a deletion of $C A T 3$ \\
\hline D.Ch.61R & cat $1 ;$ cat $2 ;$ cat $3 ;$ hygB $;$ bar & BR23-R-10 carrying a deletion of $C A T 3$ \\
\hline DCh.68R & cat $1 ;$ cat $2 ;$ cat $3 ;$ hyg $B ;$ bar & BR23-R-10 carrying a deletion of $C A T 3$ \\
\hline D.Ch.57S & cat 1 ; cat $2 ;$ hygB & Transformation of $B R 23-R-10$ \\
\hline D.Ch.64S & cat 1 ; cat $2 ;$ hygB & Transformation of $B R 23-R-10$ \\
\hline D.Ch.67S & cat 1 ; cat $2 ;$ hyg $B$ & Transformation of $B R 23-R-10$ \\
\hline
\end{tabular}


a maximum parsimony analysis with 200 random addition sequences and 200 bootstrap replicates was performed using PAUP*4.0 (Swofford 2002).

\section{Catalase gene disruptions.}

Each $C A T$ gene (CAT1, CAT2, or CAT3) gene was entirely deleted, using the split marker method described by Catlett and associates (2003), based on a modification of Fairhead and associates (1998). In the first round of PCR (Fig. 1, Tables 3 and 4$)$, the hygB cassette $(2.5 \mathrm{~kb})$ was amplified from pUCATPH (Lu et al. 1994), using M13F and M13R primers (Amberg et al. 1995). The flanking regions of CAT1, $C A T 2$, and $C A T 3$ were amplified using primer combinations CAT-P1/CAT-P2 and CAT-P3/CAT-P4 (Fig. 1, Tables 3 and 4). A second round of PCR amplified one or the other flank and about two-thirds of $\operatorname{hygB}$, as indicated in Table 3. The final PCR products ( $5^{\prime}$ flank-HY and YG-3' flank for cat 1 and cat3, 5' flank-YG and HY-3' flank for cat2) were purified using Qiagen columns (Qiagen) and were used for transformation.

Double mutants were created by crossing single mutants. PCR analysis was used to screen for deletion of pairs of catalase genes in the progeny (Fig. 1, Table 1). Triple mutant (cat1;cat2;cat3) strains D.Ch.59R, D.Ch.61R, and DCh.68R were created by deletion of CAT3 in a cat1;cat 2 double mutant (BR23-R-10), using a construct carrying the gene for resistance to bialaphos (bar, $1.5 \mathrm{~kb})$ as the selectable marker (Straubinger et al. 1990) (Table 2). For this, three rounds of PCR were performed. In the first round, the bar gene was amplified from $\mathrm{pBG}$ (Straubinger et al. 1990) using M13F/M13R. The 'primary' 5 and 3 ' flanks were amplified with primer combinations GCAT3-P0/CAT3-P2 and CAT3P3b/GCAT3-P5, respectively (Fig. 1, Tables 3 and 5). In the second round, GCAT3-P0/M13F and M13R/GCAT3-P5 were used to amplify the primary $5^{\prime}$ flank-bar and bar-primary $3^{\prime}$ flank pieces, respectively (Fig. 1). In the third round, nested primers CAT3-P1 and CAT3-P4 were used to amplify the final product (5' flank-bar-3' flank) that was used for trans- formation (Fig 1, Tables 3 and 5). The product used for transformation was purified using Qiagen columns (Qiagen).

\section{Oxidative stress assay.}

Sensitivity to oxidative stress was tested on solid MM, with or without hydrogen peroxide in petri dishes (90-mm diameter). Autoclaved $\mathrm{MM}$ was cooled to $50^{\circ} \mathrm{C}$, and then hydrogen peroxide (30\%) (Sigma) was added to a final concentration of $5 \mathrm{mM}$. Mycelial disks (3-mm diameter) were cut with a cork borer $1 \mathrm{~cm}$ from the edge of an actively growing culture. Disks were inverted and placed in the centers of plates. Single, double, and triple catalase deletion mutants were represented by three to four strains in each assay, and assays were repeated. Colony diameters on four replicate plates per strain per concentration were measured after 2 and 3 days incubation at $22^{\circ} \mathrm{C}$ in the dark. Subsequently, colony diameter increase per $24 \mathrm{~h}$ was calculated, and the data analyzed. One-way ANOVA with Bonferroni's posttest was performed using GraphPad Prism version 3.02 for Windows (GraphPad Software, San Diego, CA, U.S.A.).

Table 4. Primer combinations (primer positions in brackets) used to produce constructs for individual $C A T$ gene deletions

\begin{tabular}{ll}
\hline Primer combinations & PCR product \\
\hline First-round PCR & \\
CAT1-P3 and CAT1-P4 (D/E) & $5^{\prime}$ flank \\
CAT2-P1 and CAT2-P2 (H/I) & $5^{\prime}$ flank \\
CAT3-P1 and CAT3-P2 (N/O) & $5^{\prime}$ flank \\
CAT1-P1 and CAT1-P2 (B/C) & $3^{\prime}$ flank \\
CAT2-P3 and CAT2-P4 (J/K) & $3^{\prime}$ flank \\
CAT3-P3a and CAT3-P4 (Pa/Q) & $3^{\prime}$ flank \\
M13F and M13R & hygB cassette \\
Second-round PCR & \\
CAT1-P4 (E) and NLC 37 (HY) & $5^{\prime}$ flank-YG or -HY \\
CAT2-P1 (H) and NLC 38 (YG) & $5^{\prime}$ flank-YG or -HY \\
CAT3-P1 (N) and NLC 37 (HY) & $5^{\prime}$ flank-YG or -HY \\
NLC38 (YG) and CAT1-P1 (B) & YG- or HY-3' flank \\
NLC37 (HY) and CAT2-P4 (K) & YG- or HY-3' flank \\
NLC38 (YG) and CAT3-P4 (Q) & YG- or HY-3' flank \\
\hline
\end{tabular}

Table 3. Primers used in this study

\begin{tabular}{|c|c|c|c|}
\hline Gene & Primer location & Primer name & Sequence 5 to $3^{\prime}$ \\
\hline \multirow[t]{6}{*}{ CAT1 } & A & GCAT1-P0 & CAGAACGACGCTACACTTGA \\
\hline & B & CAT1-P1 & TCTATGCGCCAGTAGCATGA \\
\hline & $\mathrm{C}$ & CAT1-P2 & GTCGTGACTGGGAAAACCCTGGCG-TCGTTCGCACCTAGCTTTAC \\
\hline & $\mathrm{D}$ & CAT1-P3 & TCCTGTGTGAAATTGTTATCCGCT-CGCCAGAAGCAGCTCCCTTA \\
\hline & $\mathrm{E}$ & CAT1-P4 & GGCATCTGCTGGTTGGATAA \\
\hline & $\mathrm{F}$ & GCAT1-P5 & GGTGAGATGGCGGAACGATT \\
\hline \multirow[t]{6}{*}{ CAT2 } & G & GCAT2-P0 & CCAGCGACAGAAGAGGTCAA \\
\hline & $\mathrm{H}$ & CAT2-P1 & GAGTCTCGCGTGGTACATCG \\
\hline & I & CAT2-P2 & GTCGTGACTGGGAAAACCCTGGCG-AAGTCGAGTCGGCTGGCAAT \\
\hline & $\mathrm{J}$ & CAT2-P3 & TCCTGTGTGAAATTGTTATCCGCT-GAACGCCATGGTTGCATACT \\
\hline & $\mathrm{K}$ & CAT2-P4 & AGAGATGTGACGCCGACGAG \\
\hline & $\mathrm{L}$ & GCAT2-P5 & ATTGCGTCTTGGTCGACGTT \\
\hline \multirow[t]{7}{*}{ CAT3 } & $\mathrm{M}$ & GCAT3-P0 & ATCTATGCCACGCCGTACGA \\
\hline & $\mathrm{N}$ & CAT3-P1 & TGCGTGGCAAGCACAGTGTA \\
\hline & $\mathrm{O}$ & CAT3-P2 & GTCGTGACTGGGAAAACCCTGGCG-CGACGTAAGATGGCCTGGAA \\
\hline & $\mathrm{Pa}$ & CAT3-P3a & TCCTGTGTGAAATTGTTATCCGCT-CGGTCCGTTGGTGATGAATG \\
\hline & $\mathrm{Pb}$ & CAT3-P3b & TCCTGTGTGAAATTGTTATCCGCT-ATCGGTCCGTTGGTGATGAA \\
\hline & $\mathrm{Q}$ & CAT3-P4 & TTCGCCGTTGACGTCGTGAG \\
\hline & $\mathrm{R}$ & GCAT3-P5 & TGCGACGCATTGCTCAGATT \\
\hline \multirow[t]{6}{*}{ hyg $B$} & M13F & M13F & CGCCAGGGTTTTCCCAGTCACGAC \\
\hline & M13R & M13R & AGCGGATAACAATTTCACACAGGA \\
\hline & HY & $\mathrm{NLC}_{3} 7^{\mathrm{a}}$ & GGATGCCTCCGCTCGAAGTA \\
\hline & YG & NLC38 ${ }^{\mathrm{a}}$ & CGTTGCAAGACCTGCCTGAA \\
\hline & H1 & H1 & TCTCCTTGCATGCACCATTCCTT \\
\hline & $\mathrm{H} 2$ & $\mathrm{H} 2$ & TTGTGATCCGCCTGGACGACT \\
\hline \multirow[t]{2}{*}{ bar } & M13F & M13F & Discussed above \\
\hline & M13R & M13R & Discussed above \\
\hline
\end{tabular}

\footnotetext{
${ }^{a}$ Catlett et al. 2003
} 
Table 5. Primer combinations (primer positions in brackets) used to produce a construct for deletion of CAT3 from double mutant BR23-R-10 (cat $1 ;$ cat 2$)$

\begin{tabular}{ll}
\hline Primer combinations & PCR product \\
\hline First-round PCR & \\
(M/O) GCAT3-P0 and CAT3-P2 & Primary 5' flank \\
(Pb/R) CAT3-P3b and GCAT3-P5 & Primary 3' flank \\
M13F and M13R & bar cassette \\
Second-round PCR & \\
M13F and (M) GCAT3-P0 & Bar-primary 5' flank \\
GCAT3-P5 (R) and M13R & Primary 3' flank-bar \\
Third-round PCR & 5' flank-bar-3' flank \\
(N/Q) CAT3-P1 and CAT3-P4 & \\
\hline
\end{tabular}

\section{Virulence assays.}

Maize (Zea mays L.) seedlings (inbred W64A-N, 14 days old) grown in a Conviron (Winnipeg, Canada) growth chamber were inoculated by spraying with conidial suspensions in $0.05 \%$ Tween 20 harvested from 10-day-old cultures. Spore suspensions (1,000 spores per $\mathrm{ml}, 15 \mathrm{ml}$ per strain) were sprayed on five or six seedlings, using a pressurized thin layer chromatography sprayer (Alltech Associates, Deerfield, IL, U.S.A.). Inoculated seedlings were placed in a dark mist chamber for $24 \mathrm{~h}\left(22^{\circ} \mathrm{C}, 100 \%\right.$ relative humidity $)$, after which plants were transferred to a Conviron growth chamber $\left(23^{\circ} \mathrm{C}, 12-\mathrm{h}\right.$ light, and $90 \%$ relative humidity). Symptoms were evaluated five days after inoculation, and lesion sizes on the fourth oldest leaves were compared with those of wild-type strains C5 and N21-R-24. Single, double, and triple catalase deletion mutations were represented by three to four strains in each plant test. All plant tests were duplicated.

\section{ACKNOWLEDGMENTS}

This work and the sequencing of the Cochliobolus heterostrophus genome was funded by Celera Genomics for TMRI/Syngenta. The authors are grateful for the support received from S. Briggs, CEO of TMRI, and for the assistance of K. Campbell, greenhouse manager.

\section{LITERATURE CITED}

Altschul, S. F., Madden, T. L., Schaeffer, A. A., Zhang, J., Zhang, Z., Miller, W., and Lipman, D. J. 1997. Gapped BLAST and PSI-BLAST: A new generation of protein database search programs. Nucleic Acids Res. 25:3389-3402.

Amberg, D. C., Botstein, D., and Beasley, E. M. 1995. Precise gene disruption in Saccharomyces cerevisiae by double fusion polymerase chain reaction. Yeast 11:1275-1280.

Barynin, V. V., Vagin, A. A., Melik-Adamyan, V. R., Grebenko, A. I., Khangulov, S. V., Popov, A. N., Andrianova, M. E., and Vainshtein,. B. K.1986. Three-dimensional structure of T-catalase with 3 A resolution. Sov. Phys. Crystallog. 31:457-459.

Bestwick, C. S., Brown, I. R., and Mansfield, J. W. 1998. Localized changes in peroxidase activity accompany hydrogen peroxide generation during the development of non-host hypersensitive reaction in lettuce. Plant Physiol 118: 1067-1078.

Bussink, H. J., and Oliver R. 2001. Identification of two highly divergent catalase genes in the fungal tomato pathogen, Cladosporium fulvum. Eur. J. Biochem. 268:15-24.

Calera, J. A., Paris, S., Monod, M., Hamilton, A. J., Debeaupuis, J. P., Diaquin, M., Lopez-Medrano, R., Leal, F., and Latge, J. P. 1997. Cloning and disruption of the antigenic catalase gene of Aspergillus fumigatus. Infect. Immun. 65:4718-4724.

Catlett, N. L., Lee, B.-N., Yoder, O. C., and Turgeon, B. G. 2003. Splitmarker recombination for efficient targeted deletion of fungal genes. Fungal Genet. Newsl. 50: 9-11.

Drechsler, C. 1925. Leafspot of maize caused by Ophiobolus heterostrophus $\mathrm{n}$. sp. the ascigerous stage of a Helminthosporium exhibiting bipolar germination. J. Agr. Res. 31:701-726.

Fairhead, C., Thierry, A., Denis, F., Eck, M., and Dujon, B. 1998. 'Massmurder' of ORFs from three regions of chromosome XI from Saccharomyces cerevisiae. Gene 223:33-46.
Fraaije, M. W., Roubroeks, H. P., Hagen, W. R., and Van Berkel, W. J. H. 1996. Purification and characterization of an intracellular catalaseperoxidase from Penicillium simplicissium. Eur. J. Biochem. 235:192198.

Garre, V., Muller, U., and Tudzynski, P. 1998. Cloning, characterization, and targeted disruption of cpcat1, coding for an in planta secreted catalase of Claviceps purpurea. Mol. Plant Microbe Interact. 11:772783.

Gould, S. J., Keller, G. A., Hosken, N., Wilkinson, J., and Subramani, S. 1989. A conserved tripeptide sorts proteins to peroxisomes. J. Cell Biol. 108:1657-1664.

Huelsenbeck, J. P., and Ronquist, F. 2001. MRBAYES: Bayesian inference of phylogenetic trees. Bioinformatics 17:754-755.

Kawasaki, L., and Aguirre, J. 2001. Multiple catalase genes are differentially regulated in Aspergillus nidulans. J Bacteriol. 183:1434 1440 .

Keissar, H. T., Bashan, B., Levy, Y., and Kenigsbuch, D. 2002. Stage specificity of catalase isoform activity in Exserohilum turcicum. Physiol. Mol. Plant Path. 60:163-168.

Keppler, L. D., Baker, C. J., and Atkinson, M. M. 1989. Active oxygen production during a bacteria induced hypersensitive reaction in tobacco suspension cells. Phytopathology 79:974-978.

Klotz, M. G., Klassen, G. R., and Loewen, P. C. 1997. Phylogenetic relationships among prokaryotic and eukaryotic catalases. Mol. Biol. Evol. 14:951-958.

Kumar, J., Hückelhoven, R., Beckhove, U., Nagarajan, S., and Kogel, K.-H. 2001. A compromised Mlo Pathway affects the response of barley to the necrotrophic fungus Bipolaris sorokiniana (teleomorph: Cochliobolus sativus) and its toxins. Phytopathology 91:127-133.

Leach, J., Lang, B. R., and Yoder, O. C. 1982. Methods for selection of mutants and in vitro culture of Cochliobolus heterostrophus. J. Gen. Microbiol. 128:1719-1729.

Lu, S., Lyngholm, L., Yang, G., Bronson, C., Yoder, O. C., and Turgeon, B. G. 1994. Tagged mutations at the Tox1 locus of Cochliobolus het erostrophus by restriction enzyme-mediated integration. Proc. Natl. Acad. Sci. U.S.A. 91:12649-12653.

Mehdy, M. C. 1994. Active oxygen species in plant defense against pathogens. Plant Physiol. 105:467-472.

Nicholas, K. B., Nicholas, H. B., and Deerfield, D. W. 1997. GeneDoc: Analysis and visualization of genetic variation. Embnew.News 4:14

Nielsen, H., Engelbrecht, J., Brunak, S., and von Heijne, G. 1997. Identification of prokaryotic and eukaryotic signal peptides and prediction of their cleavage sites. Protein Eng. 10:1-6.

Notredame, C., Higgins, D. G., and Heringa, J. 2000. T-Coffee: A novel method for fast and accurate multiple sequence alignment. J. Mol. Biol 302:205-217.

Rutherford, K., Parkhill, J, J., Crook, J., Horsnell, T., Rice, P., Rajandream, M.-A., and Barrell, B. 2000. Artemis: Sequence visualisation and annotation. Bioinformatics 16:944-945.

Scherer, M., Wei, H., Liese, R., and Fischer, R. 2002. Aspergillus nidulans catalase-peroxidase gene (cpeA) is transcriptionally induced during sexual development through the transcription factor StuA. Eucaryotic Cell 1:725-735.

Schonbaum, G. R., and Chance, B. 1976. Catalase. Pages 863-408 in: The Enzymes. P. D. Boyer, ed. Academic Press. New York.

Schouten, A., Tenberge, K. B., Vermeer, J., Stewart, J., Wagemakers, L., Williamson, B., and Van Kan, J. A. L. 2002. Functional analysis of an extracellular catalase of Botrytis cinerea. Mol. Plant Pathol. 3:227-238.

Shim, W.-B., and L. D. Dunkle. 2002. Identification of genes expressed during cercosporin biosynthesis in Cercospora zeae-maydis. Physiol. Mol. Plant Pathol. 61:237-248.

Straubinger, B., Straubinger, E., Wirsel, S., Turgeon, B. G., and Yoder, O. 1990. Versatile fungal transformation vectors carrying the selectable bar gene of Streptomyces hygroscopicus. Fungal Genet. Newsl. 39:82-83.

Swinkels, B. W., Gould, S. J., Bodnar, A. G., Rachubinski, R. A., and Subramani, S. 1991. A novel, cleavable peroxisomal targeting signla at the amino-terminus of the rat 3-ketoacyl-CoA thiolase. EMBO (Eur. Mol. Biol. Organ.) J. 10:3255-3262.

Swofford, D. L. 2002. Phylogenetic analysis using parsimony (*and other methods). Sinauer Associates, Sunderland, MA, U.S.A.

Thompson, J. E., Legge, R. L., and Barber, R. F. 1987. The role of free radicals in senescence and wounding. New Phytol. 105:317-344.

Turgeon, B. G., Garber, R. C., and Yoder, O. C. 1987. Development of a fungal transformation system based on selection of sequences with promoter activity. Mol. Cell. Biol. 7:3297-3305.

Tzeng, T. H., Lyngholm, L. K., Ford, C. F., and Bronson, C. R. 1992. A restriction fragment length polymorphism map and electrophoretic 
karyotype of the fungal maize pathogen Cochliobolus heterostrophus. Genetics 130:81-96.

Wang, H., Li, J., Bostock, R. M., and Gilchrist, D. G. 1996. Apoptosis: A functional paradigm for programmed plant cell death induced by a host-selective phytotoxin and invoked during development. Plant Cell 8:375-391.

Wirsel, S., Turgeon, B. G., and Yoder, O. C. 1996. Deletion of the Cochliobolus heterostrophus mating-type (MAT) locus promotes the function of MAT transgenes. Curr. Genet. 29:241-249.
Wysong, D. R., Christin, L., Sugar, A. M., Robbins, P. W., and Diamond, R. D. 1998. Cloning and sequencing of a Candida albicans catalase gene and effects of disruption of this gene. Infect. Immun. 66:1953-1961.

AUTHOR-RECOMMENDED INTERNET RESOURCE

GraphPad software website: www.graphpad.com 